\title{
GENETIC EVALUATION OF CROSSBRED CATTLE FOR
}

\section{REPRODUCTION TRAITS}

\section{SUNIL KUMAR, D. S. DALAL, B. L. PANDER, C. S. PATIL \& RAKESH VERMA \\ Department of Animal Genetics and Breeding, Lala Lajpat Rai University of}

Veterinary and Animal Sciences, Hisar-, India

\begin{abstract}
The data on 333 crossbred cows maintained in the Department of Animal Genetics and Breeding, Lala Lajpat Rai University of Veterinary and Animal Sciences, Hisar, Haryana over a period of 24 years from 1991 to 2014 were analyzed for studying the effect of genetic factor i.e. sire group and non genetic factors i.e. period of calving, season of calving and milk yield group and to estimate genetic and phenotypic parameters for reproduction traits. The traits studied were age at first calving (AFC), first service period (FSP), first calving interval (FCI), number of services per first conception (NS/FCON), waiting period (WP) and daughter pregnancy rate (DPR42, DPR63). The least squares mean for AFC, FSP, FCI, NS/FCON, WP, DPR42 and DPR63 were observed as $1242.75 \pm 16.46$ days, $148.46 \pm 6.99$ days, $429.08 \pm 7.08$ days, $1.90 \pm 0.12,100.85 \pm 4.20$ days, $0.27 \pm 0.02$ and $0.35 \pm 0.04$, respectively. The period of calving had significant effect on AFC, while season of calving had significant effect on FSP and FCI. The milk yield group had significant effect on all traits. Heritability estimates of these traits were low to moderate. Genetic and phenotypic correlations among FSP, FCI, WP and NS/FCON were found to be moderate to high positive. DPR showed moderate and negative genetic correlation with FSP, FCI, WP and NS/FCON whereas; corresponding phenotypic correlations with these traits were significant negative.

KEYWORDS: Correlation, Crossbred Cattle, Daughter Pregnancy Rate, Heritability \& Reproduction Traits
\end{abstract}

Received: Oct 13, 2017; Accepted: Nov 03, 2017; Published: Nov 15, 2017; Paper Id.: IJASRDEC201729

\section{INTRODUCTION}

The economic worth of milch animals is primarily determined by their productive performances. Hence in selection process, generally more emphasis is given to production traits. In past decades successful selection on milk production traits in dairy cattle has led to decline in female fertility due to unfavorable, correlated selection response (Jorjani, 2006). Thus, incorporation of fertility in selection decisions seems desirable. Improvement of fertility in lactating animals has become the key objective for selection programs in recent years in most countries. Many countries have used Daughter Pregnancy Rate and Service period for genetic evaluation of their dairy animals.

It is essential to have knowledge of genetic and non-genetic factors influencing the performance traits to obtain unbiased estimates of genetic parameters and for developing a suitable selection criterion. The accuracy of selection and genetic correlation between performance traits contribute much to the success of any breeding programme. Therefore, the present investigation was carried out to estimate genetic and phenotypic parameters of different first lactation reproduction traits and to study the effect of genetic and non-genetic factors on these traits. 


\section{MATERIALS AND METHODS}

The first lactation records of 333 crossbred cattle maintained in the Department of Animal Genetics and Breeding, LUVAS, Hisar over the period of 24 years from 1991 to 2014 were utilised for the present study. The traits under study were age at first calving (AFC), first service period (FSP), first calving interval (FCI), number of services per first conception (NS/FCON), waiting period (WP) and daughter pregnancy rate (DPR42, DPR63). Abnormal lactation records due to specific causes like abortion and sickness were excluded. The entire duration of 24 years from 1991 to 2014 was divided into 8 periods each having three years duration. Year to year variation within the period was assumed to be non-significant. Each year was divided into four seasons viz. summer (April to June), rainy (July to Sept.), autumn (Oct. to Nov.) and winter (Dec. to March) on the basis of fluctuations in atmospheric temperature and relative humidity.

The waiting period or days to first service is the initial phase of lactation during which no insemination occurs. WP = (DOFAI - DOFC) Where, DOFAI- date of first AI after first calving and DOFC- date of first calving. Daughter pregnancy rate measures how quickly cows become pregnant again after calving. It is defined as the percentage of non pregnant cows that become pregnant during each 21 days period, because each estrus cycle represents one chance for an animal to become pregnant. DPRs were estimated as suggested by USDA (2003). DPR $=21$ / (First Service Period - Voluntary Waiting Period + 11). Daughter Pregnancy rate (DPR 42, 63) for crossbred cattle were estimated as: DPR $(42)=21 /($ First Service Period - Waiting Period $(42)+11) ;$ DPR $(63)=21 /($ First Service Period - Waiting Period $(63)+11)$.

To study the effect of genetic factor i.e. sire group and non-genetic factors i.e. period of calving, season of calving and milk yield group on different reproduction traits and to obtain sire and residual variance-covariance components for various reproduction traits, least squares analysis technique (Harvey, 1990) was used with the following mixed model:

$$
Y_{i j k l m n}=\mu+G_{i}+T_{i j}+P_{k}+S_{l}+M Y_{m}+b\left(A_{i j k l m n}-\bar{A}\right)+e_{i j k l m n}
$$

Where,

$\mathrm{Y}_{\mathrm{ijklmn}}=$ Observation of $\mathrm{n}^{\text {th }}$ progeny of $\mathrm{j}^{\text {th }}$ sire belonging to $\mathrm{i}^{\text {th }}$ sire group, $\mathrm{k}^{\text {th }}$ period of calving, $\mathrm{l}^{\text {th }}$ season of calving and $\mathrm{m}^{\text {th }}$ milk yield group,

$\mu \quad=$ Overall population mean,

$\mathrm{G}_{\mathrm{i}}=$ Fixed effect of $\mathrm{i}^{\text {th }}$ sire group,

$\mathrm{T}_{\mathrm{ij}}=$ Random effect of $\mathrm{j}^{\text {th }}$ sire within $\mathrm{i}^{\text {th }}$ sire group,

$\mathrm{P}_{\mathrm{k}}=$ Fixed effect of $\mathrm{k}^{\text {th }}$ period of calving $(\mathrm{k}=1$ to 8$)$,

$\mathrm{S}_{1}=$ Fixed effect of $\mathrm{l}^{\text {th }}$ season of calving $(1=1,2,3,4)$,

$\mathrm{MY}_{\mathrm{m}}=$ Fixed effect of $\mathrm{m}^{\text {th }}$ milk yield group $(1=1,2,3,4)$,

b = Linear regression coefficient of a trait on age at first calving,

$\mathrm{A}_{\mathrm{ijklmn}}=$ Age at first calving pertaining to $\mathrm{Y}_{\mathrm{ijklmn}}{ }^{\text {th }}$ observation,

$\overline{\mathrm{A}}=$ Mean age at first calving,

$\mathrm{e}_{\mathrm{ijklmn}}=$ Random residual error associated with observation of $n^{\text {th }}$ progeny of $\mathrm{j}^{\text {th }}$ sire belonging to $\mathrm{i}^{\text {th }}$ sire 
group, $\mathrm{k}^{\text {th }}$ period of calving, $\mathrm{l}^{\text {th }}$ season of calving and $\mathrm{m}^{\text {th }}$ milk yield group assumed to be normally and independently distributed with mean zero and variance $\sigma_{\mathrm{e}}^{2}$.

To study AFC the above model was used after deleting the effect of AFC as covariate.

Genetic and phenotypic parameters for different reproduction traits were obtained by paternal half-sib correlation method as per standard procedure. The standard errors of phenotypic correlations were computed by using the formula given by Snedecor and Cochran (1968).

\section{RESULTS AND DISCUSSIONS}

\section{Least-Squares Mean for Different Reproduction Traits}

The overall least squares mean for AFC was found to be $1242.75 \pm 16.46$ days. (Table 1) This estimate was supported by the findings of Chaudhari et al. (2013), Goshu et al. (2014) and Kumar (2015). The AFC obtained in the present study is in lower side than those reported by Singh et al. (2008) and Hassan and Khan (2013) in crossbred cattle. While, Saha et al. (2010) and Divya (2012) reported lower AFC than the present study. The overall least squares mean for first service period (148.46 \pm 6.99 days) reported in the present study is in analogy with the findings of Singh and Gurnani (2004) and Raja and Gandhi (2015). However, lower FSP than the present study were reported by Saha et al. (2010) and Kumar (2015) in crossbred cattle. The estimates reported by Hassan and Khan (2013) and Goshu et al. (2014) were on the higher side.

The overall least squares mean of FCI was $429.08 \pm 7.08$ days in the present study. The results for FCI were comparable with those reported by Saha et al. (2010), Chaudhari et al. (2013) and Kumar (2015) in crossbred cattle. Divya (2012) reported lower FCI than the present study, whereas, higher FCI was reported by Hassan and Khan (2013) and Goshu et al. (2014). The overall least squares mean of NS/FCON was $1.90 \pm 0.12$. The present results for NS/FCON are comparable with those reported by Dematawewa and Berger (1998) and Veerkamp et al. (2001) whereas, Grosshans et al. (1997) reported lower NS/FCON than the present study.

The overall least squares mean of WP was $100.85 \pm 0.12$ days in the present study. This estimate is in close conformity with that reported by Hammoud et al. (2010). Grosshans et al. (1997) reported lower WP than the present study. The least squares means for DPR42 and DPR63 were observed as $0.27 \pm 0.02$ and $0.35 \pm 0.04$, respectively.

\section{Effect of Genetic and non Genetic Factors on Different Reproduction Traits Effect of Sire Group}

The influence of sire group was non-significant on all first lactation reproduction traits except AFC (Table 2). Kumar (2015) also reported non-significant effect of sire group on FSP and FCI. Divya (2012) supported significant effect of sire group on AFC.

\section{Effect of Period of Calving}

Period of calving had highly significant effect on AFC which is in agreement with the observations made by Divya (2012), Chaudhari et al. (2013) and Kumar (2015) in crossbred cattle. The variation due to period reflects managerial differences, variation in availability of quality and quantity of feed and fodders during different periods. 


\section{Effect of Season of Calving}

Analysis of variance (Table 2) showed that the influence of season of calving was highly significant $(\mathrm{p}<0.01)$ on FSP and FCI. Saha et al. (2010) also reported significant effect of season of calving on these traits. Non-significant effect of season of calving on WP and DPR was supported by Divya (2012). Autumn calvers showed better reproductive performance than the animals calved in other seasons. It might be due to better availability of feed and fodder and better adaptability of crossbred cattle to favourable climate.

\section{Effect of AFC}

Effect of AFC was significant $(\mathrm{p}<0.05)$ on FSP and FCI while it was non-significant on other traits. However, Kumar (2015) reported non-significant effect of AFC on these traits.

\section{Effect of Milk Yield Group}

Milk yield group had significant effect on all reproduction traits. The results showed that high yielder animals were reproductively inferior indicating existence of antagonistic relationship between production and fertility.

\section{Heritability Estimates}

The estimates of heritability for reproduction traits were low to moderate (Table 3). The heritability estimate of AFC $(0.38 \pm 0.14)$ is in conformity with heritability estimate reported by Kumar et al. (2008) in crossbred cattle. While, higher estimate of heritability were reported by Chaudhari et al. (2013) and Ghosu et al. (2014). The estimate of heritability for FSP was moderate (0.22 \pm 0.22$)$. Similar results were reported by Goshu et al. (2014) in Holstein-Friesian cattle. However, higher estimates of heritability of FSP were reported by Chaudhari et al. (2013). The heritability estimate of FCI was also moderate $(0.28 \pm 0.19)$. Similar magnitudes of heritability were reported by Saha et al. (2010) and Goshu et al.

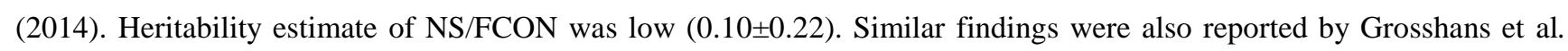
(1997) and Veerkamp et al. (2001). The heritability of WP was moderate. However, Grosshans et al. (1997) and Veerkamp et al. (2001) reported lower estimates of heritability for WP. Heritability estimate of DPR42 and DPR63 were low $(0.11 \pm 0.18$ and $0.13 \pm 0.01$, respectively). VanRaden et al. (2004) also reported low heritability estimates of DPR. The high environmental variance in the reproductive traits might be reason for low heritability. So, the improvement in these traits could be achieved by effective implementation of corrective measures of managemental practices.

\section{Genetic and Phenotypic Correlations}

Genetic and phenotypic correlations among different reproduction traits are presented in Table 3 . The AFC was found to have low genetic and phenotypic correlation with all traits except NS/FCON. The estimates of genetic correlations have low reliability due to high standard error. The results of phenotypic correlations of AFC with FSP and FCI were in agreement to those reported by Chaudhari et al. (2013) and Goshu et al. (2014). WP had high positive genetic and phenotypic correlation with FSP and FCI and negative correlations with NS/FCON. Similar findings were also reported by Grosshans et al. (1997). NS/FCON also had high genetic and phenotypic correlation with FSP and FCI. Grosshans et al. (1997) and Dematawewa and Berger (1998) supported these findings. High positive genetic and phenotypic correlations among FSP and FCI were supported by Chaudhari et al. (2013), Goshu et al. (2014) and Kumar (2015).

Genetic and phenotypic correlations between DPR42 and DPR63 were high and significant. Patil (2011) also reported high genetic and phenotypic correlations among various DPR. DPRs showed moderate to high negative genetic 
correlation with FSP, FCI, WP and NS/FCON whereas; corresponding phenotypic correlations with these traits were significant negative. Genetic correlations among most of the reproduction traits were high which is expected because of their biological relationship and most of these traits could be expressed as a function of another trait (Kadarmideen et al., 2003).

\section{CONCLUSIONS}

Heritability estimates for reproduction traits were low to moderate. So, the improvement in the reproduction traits could be achieved by better managemental practices. High positive genetic correlations among reproduction traits indicated that animals superior in one trait would perform better in other traits as well. So, it could be expected that genetic improvement of one reproduction trait will automatically cause improvement in the other traits as well.

\section{REFERENCES}

1. Chaudhari, M., Kumar, R., Khanna, A. S., Dalal, D. S. \& Jakhar, A. (2013). Genetic studies on reproduction traits in crossbred cattle. Haryana Vet., 52,75-78.

2. Dematawewa, C. M. B. \& Berger, P. J. (1998). Genetic and phenotypic parameters for 305-day yield, fertility, and survival in Holsteins. J. Dairy Sci., 81(10), 2700-2709.

3. Divya, P. (2012). Single versus multi-trait models for genetic evaluation of fertility traits in Karan Fries cattle. M. V. Sc. Thesis, NDRI (Deemed University) Karnal, India

4. Goshu, G., Singh, H., Peterson, K. J. \& Lundeheim, N. (2014). Heritability and correlation among first lactation traits in Holstein Friesian cows at Holeta bull dam station, Ethiopia. Int. J. Livest. Prod., 5, 47-53

5. Grosshans, T., Xu, Z. Z., Burton, L. J., Johnson, D. L. and Macmillan, K. L. (1997). Performance and genetic parameters for fertility of seasonal dairy cows in New Zealand. Livest. Prod. Sci., 51(1), 41-51.

6. Hammoud, M. H., El-Zarkouny, S. Z. \& Oudah, E. Z. M. (2010). Effect of sire, age at first calving, season and year of calving and parity on reproductive performance of Friesian cows under semiarid conditions in Egypt. Arch. zootech. 13, 60-82.

7. Harvey, W. R. (1990). Guide for LSMLMW, PC-1 Version, mixed model least squares and maximum likelihood computer programme, January 1990. Mimeograph Ohio State Univ., USA.

8. Hassan, F. \& Khan, M. S. (2013). Performance of crossbred dairy cattle at military dairy farms in Pakistan. J. Anim. Plant Sci., 23(3), 705-714.

9. Jorjani, H. (2006). International genetic evaluation for female fertility traits. Interbull Bull. 35: $42-46$.

10. Kadarmideen, H.N., Thompson, R., Coffey, M.P. \& Kossaibati, M.A., (2003). Genetic parameters and evaluations from singleand multiple-trait analysis of dairy cow fertility and milk production. Livest. Prod. Sci., 81(2), 183-195.

11. Kumar, P. (2015). Genetic evaluation of Frieswal sires for test day milk records and first lactation traits. M.V.Sc. Thesis, LUVAS, Hisar, India.

12. Kumar, S., Singh, Y.P. \& Kumar, D. (2008). Genetic studies on performance traits in Frieswal cattle. Ind. J. Anim. Sci., 78, 107-110.

13. Patil, C.S. (2011). Genetic evaluation of fertility in Murrah buffaloes. M.V.Sc. Thesis. NDRI (Deemed University), Karnal, India. 
14. Raja, T.V. \& Gandhi, R.S. (2015). Factors influencing productive and reproductive performance of Sahiwal cattle maintained at organized farm conditions. Ind. J. Anim. Sci., 85(6).

15. Saha, S., Joshi, B.K. \& Singh, A. (2010). Generation-wise genetic evaluation of various first lactation traits and herd life in Karan Fries cattle. Ind. J. Anim. Sci., 80: 451-456

16. Singh, M.K. \& Gurnani, M. (2004). Genetic analysis of production and reproduction traits in Karan-Fries and Karan-Swiss cattle. Ind. J. Anim. Sci., 74: 225-228.

17. Singh, V.K., Singh, C.V., Kumar, D. \& Sharma, R.J. (2008). Genetic parameter for first lactation and life time performance traits in crossbred cattle. Ind. J. Anim. Sci., 78, 497-500

18. Snedecor, G.W. \& Cochran, W.G. (1968). Statistical methods (6 ${ }^{\text {th }}$ edn.) Oxford and IBH publishing Co., New Delhi.

19. USDA. (2003). Estimated relative conception rate evaluation. Animal Improvement Programs Laboratory. (www.google.com)

20. VanRaden, P.M., Sandres, A.H., Tooker, M.E., Miller, R.H., Norman, H.D., Kuhn,H.T. \& Wiggans, G.R (2004). Development of national genetic evaluation for cow fertility. J. Dairy Sci., 87, 2285-2292.

21. Veerkamp, R.F., Koenen, E.P.C., \& De Jong, G. (2001). Genetic correlations among body condition score, yield, and fertility in first-parity cows estimated by random regression models. J. Dairy Sci., 84(10), 2327-2335.

Table 1: Least Squares Means Along with Standard Errors for Different Reproduction Traits

\begin{tabular}{|c|c|c|c|c|c|c|c|c|}
\hline \multirow{2}{*}{\multicolumn{2}{|c|}{ Effect }} & \multicolumn{7}{|c|}{ Least Squares Mean \pm S.E. } \\
\hline & & AFC (days) & FSP (days) & FCI (days) & WP (days) & NS/FCON & DPR42 & DPR63 \\
\hline \multicolumn{2}{|c|}{$\begin{array}{l}\text { OVERALL } \\
\text { MEAN }\end{array}$} & $\begin{array}{c}1242.75 \pm 16.46 \\
(333)\end{array}$ & $\begin{array}{c}148.46 \pm 6.99 \\
(333)\end{array}$ & $\begin{array}{c}429.08 \pm 7.08 \\
(333)\end{array}$ & $\begin{array}{c}100.85 \pm 4.2 \\
0(333)\end{array}$ & $\begin{array}{c}1.90 \pm 0.12 \\
(333)\end{array}$ & $\begin{array}{c}0.27 \pm 0.02 \\
(318)\end{array}$ & $\begin{array}{c}0.35 \pm 0.04 \\
(245)\end{array}$ \\
\hline \multirow{8}{*}{$\begin{array}{c}\text { Sire } \\
\text { group }\end{array}$} & SG 1 & $\begin{array}{c}1136.01^{\mathrm{a}} \pm 138.5 \\
9(50)\end{array}$ & $\begin{array}{c}196.68 \pm 55.0 \\
7(50) \\
\end{array}$ & $\begin{array}{c}472.23 \pm 55.5 \\
8(50)\end{array}$ & $\begin{array}{c}109.49 \pm 34 . \\
98(50)\end{array}$ & $\begin{array}{c}1.86 \pm 1.02 \\
(50)\end{array}$ & $\begin{array}{c}0.25 \pm 0.23 \\
(49)\end{array}$ & $\begin{array}{c}0.49 \pm 0.33 \\
(41) \\
\end{array}$ \\
\hline & SG 2 & $\begin{array}{c}1181.47^{\mathrm{ab}} \pm 105 \\
12(11)\end{array}$ & $\begin{array}{c}146.43 \pm 43.7 \\
0(11)\end{array}$ & $\begin{array}{c}427.25 \pm 44.2 \\
1(11)\end{array}$ & $\begin{array}{c}101.86 \pm 26 . \\
92(11)\end{array}$ & $\begin{array}{l}1.61 \pm 0.80 \\
\quad(11)\end{array}$ & $\begin{array}{c}0.30 \pm 0.18 \\
(11)\end{array}$ & $\begin{array}{l}0.56 \pm 0.27 \\
\quad(10)\end{array}$ \\
\hline & SG 3 & $\begin{array}{c}1396.51^{\mathrm{e}} \pm 91.58 \\
(26)\end{array}$ & $\begin{array}{c}130.09 \pm 36.2 \\
2 \\
(26)\end{array}$ & $\begin{array}{c}410.76 \pm 36.5 \\
1 \\
(26)\end{array}$ & $\begin{array}{c}98.82 \pm 23.4 \\
6 \\
(26)\end{array}$ & $\begin{array}{l}1.58 \pm 0.68 \\
(26)\end{array}$ & $\begin{array}{c}0.20 \pm 0.15 \\
(26)\end{array}$ & $\begin{array}{l}0.53 \pm 0.22 \\
\quad(24)\end{array}$ \\
\hline & SG 4 & $\begin{array}{c}1260.69^{c} \pm 65.74 \\
(46)\end{array}$ & $\begin{array}{c}153.13 \pm 28.3 \\
3 \\
(46)\end{array}$ & $\begin{array}{c}436.45 \pm 28.7 \\
5 \\
(46)\end{array}$ & $\begin{array}{c}101.81 \pm 16 . \\
57 \\
(46)\end{array}$ & $\begin{array}{l}2.14 \pm 0.50 \\
\quad(46)\end{array}$ & $\begin{array}{c}0.24 \pm 0.11 \\
\quad(43)\end{array}$ & $\begin{array}{c}0.30 \pm 0.17 \\
(37)\end{array}$ \\
\hline & SG 5 & $\begin{array}{c}1255.10^{\mathrm{c}} \pm 67.61 \\
(90)\end{array}$ & $\begin{array}{c}132.36 \pm 29.1 \\
7(90)\end{array}$ & $\begin{array}{c}417.28 \pm 29.6 \\
1(90)\end{array}$ & $\begin{array}{c}96.36 \pm 17.0 \\
5(90)\end{array}$ & $\begin{array}{c}1.96 \pm 0.51 \\
(90)\end{array}$ & $\begin{array}{c}0.31 \pm 0.11 \\
(84)\end{array}$ & $\begin{array}{c}0.27 \pm 0.18 \\
(58)\end{array}$ \\
\hline & SG 6 & $\begin{array}{c}1325.17^{\mathrm{d}} \pm 77.28 \\
(27)\end{array}$ & $\begin{array}{c}143.58 \pm 32.3 \\
1(27) \\
\end{array}$ & $\begin{array}{c}426.99 \pm 32.7 \\
3(27) \\
\end{array}$ & $\begin{array}{c}108.32 \pm 19 . \\
47(27)\end{array}$ & $\begin{array}{c}1.79 \pm 0.58 \\
(27)\end{array}$ & $\begin{array}{c}0.27 \pm 0.13 \\
(27)\end{array}$ & $\begin{array}{c}0.12 \pm 0.20 \\
(20)\end{array}$ \\
\hline & SG 7 & $\begin{array}{c}1233.18^{\mathrm{bc}} \pm 77.7 \\
3 \\
(51)\end{array}$ & $\begin{array}{c}152.22 \pm 34.4 \\
0(51)\end{array}$ & $\begin{array}{c}434.05 \pm 34.9 \\
6(51)\end{array}$ & $\begin{array}{c}109.45 \pm 19 . \\
64(51)\end{array}$ & $\begin{array}{l}2.00 \pm 0.60 \\
(51)\end{array}$ & $\begin{array}{c}0.29 \pm 0.13 \\
(47)\end{array}$ & $\begin{array}{l}0.14 \pm 0.21 \\
\quad(38)\end{array}$ \\
\hline & SG 8 & $\begin{array}{c}1153.83^{\mathrm{a}} \pm 80.37 \\
\text { (32) }\end{array}$ & $\begin{array}{c}133.17 \pm 32.1 \\
1 \\
(32) \\
\end{array}$ & $\begin{array}{c}407.61 \pm 32.4 \\
1 \\
(32) \\
\end{array}$ & $\begin{array}{c}80.69 \pm 20.4 \\
3 \\
(32) \\
\end{array}$ & $\begin{array}{l}2.30 \pm 0.60 \\
\quad(32)\end{array}$ & $\begin{array}{l}0.32 \pm 0.14 \\
\quad(31)\end{array}$ & $\begin{array}{c}0.41 \pm 0.19 \\
\quad(17)\end{array}$ \\
\hline \multirow{3}{*}{$\begin{array}{l}\text { Milk } \\
\text { yield } \\
\text { group }\end{array}$} & $\mathbf{I}$ & - & $\begin{array}{c}116.87^{\mathrm{a}} \pm 10 \\
11(75)\end{array}$ & $\begin{array}{c}396.28^{\mathrm{a}} \pm 10 \\
15(75)\end{array}$ & $\begin{array}{c}90.87^{\mathrm{a}} \pm 6.8 \\
9 \\
(75)\end{array}$ & $\begin{array}{c}1.58^{\mathrm{a}} \pm 0.19 \\
(75)\end{array}$ & $\begin{array}{c}0.36^{\mathrm{b}} \pm 0.0 \\
4 \\
(72)\end{array}$ & $\begin{array}{c}0.45^{\mathrm{b}} \pm 0.0 \\
6 \\
(54)\end{array}$ \\
\hline & II & - & $\begin{array}{c}131.67^{\mathrm{ab}} \pm 9.4 \\
7 \\
(99)\end{array}$ & $\begin{array}{c}410.25^{\mathrm{ab}} \pm 9.5 \\
2 \\
(99)\end{array}$ & $\begin{array}{c}97.96^{\mathrm{a}} \pm 6.3 \\
6 \\
(99)\end{array}$ & $\begin{array}{c}1.72^{\mathrm{ab}} \pm 0.18 \\
\quad(99)\end{array}$ & $\begin{array}{c}0.34^{\mathrm{b}} \pm 0.0 \\
4 \\
(97)\end{array}$ & $\begin{array}{c}0.29^{\mathrm{a}} \pm 0.0 \\
6 \\
(75)\end{array}$ \\
\hline & III & - & $\begin{array}{c}149.59^{\mathrm{b}} \pm 10 \\
23(76)\end{array}$ & $\begin{array}{c}432.88^{\mathrm{b}} \pm 10 \\
27 \\
(76)\end{array}$ & $\begin{array}{c}98.47^{\mathrm{a}} \pm 6.9 \\
9 \\
(76)\end{array}$ & $\begin{array}{c}2.06^{\mathrm{bc}} \pm 0.20 \\
(76)\end{array}$ & $\begin{array}{c}0.27^{\mathrm{b}} \pm 0.0 \\
4 \\
(71)\end{array}$ & $\begin{array}{c}0.45^{\mathrm{b}} \pm 0.0 \\
6 \\
(55)\end{array}$ \\
\hline
\end{tabular}




\begin{tabular}{|c|c|c|c|c|c|c|c|c|}
\hline & \multicolumn{10}{|c|}{ Table 1: Contd., } \\
\cline { 2 - 8 } & \multirow{2}{*}{ IV } & & $195.68^{\mathrm{c}} \pm 10$. & $476.89^{\mathrm{c}} \pm 10$. & $116.10^{\mathrm{b}} \pm 7$. & $2.26^{\mathrm{c}} \pm 0.20$ & $0.12^{\mathrm{a}} \pm 0.0$ & $0.22^{\mathrm{a}} \pm 0.0$ \\
& & - & 59 & 62 & 28 & 5 & 7 \\
$(83)$ & $(83)$ & $(83)$ & $(83)$ & $(83)$ & $(78)$ & $(61)$ \\
\hline
\end{tabular}

\begin{tabular}{|c|c|c|c|c|c|c|c|c|}
\hline \multirow{2}{*}{\multicolumn{2}{|c|}{ Effect }} & \multicolumn{7}{|c|}{ Least Squares Mean \pm S.E. } \\
\hline & & \multirow{2}{*}{$\begin{array}{c}\begin{array}{c}\text { AFC } \\
\text { (Days) }\end{array} \\
1316.44^{\mathrm{c}} \pm 1 \\
50.24(44) \\
\end{array}$} & \multirow{2}{*}{$\begin{array}{c}\text { FSP (Days) } \\
249.67 \pm 79.92 \\
(44)\end{array}$} & \multirow{2}{*}{$\begin{array}{c}\text { FCI (Days) } \\
501.25 \pm 79.5 \\
5(44)\end{array}$} & \multirow{2}{*}{$\begin{array}{c}\text { WP (Days) } \\
\begin{array}{c}131.10 \pm 59.69 \\
(44)\end{array}\end{array}$} & \multirow{2}{*}{$\begin{array}{c}\mathbf{N S} / \mathrm{FCO} \\
\mathbf{N} \\
\begin{array}{c}2.92 \pm 1.66 \\
(44)\end{array} \\
\end{array}$} & \multirow{2}{*}{$\begin{array}{c}\text { DPR42 } \\
0.06 \pm 0.40 \\
(43)\end{array}$} & \multirow{2}{*}{$\begin{array}{c}\text { DPR63 } \\
0.05 \pm 0.4 \\
7 \\
(35) \\
\end{array}$} \\
\hline \multirow{8}{*}{$\begin{array}{c}\text { Period } \\
\text { of } \\
\text { calving }\end{array}$} & P1(1991-93) & & & & & & & \\
\hline & P2(1994-96) & $\begin{array}{c}1443.84^{\mathrm{d}} \pm 1 \\
33.19 \\
(10) \\
\end{array}$ & $\begin{array}{c}203.30 \pm 67.27 \\
(10)\end{array}$ & $\begin{array}{c}488.37 \pm 66.9 \\
7(10)\end{array}$ & $\begin{array}{c}131.02 \pm 50.02 \\
(10)\end{array}$ & $\begin{array}{l}1.87 \pm 1.40 \\
(10)\end{array}$ & $\begin{array}{c}0.01 \pm 0.33 \\
\quad(10)\end{array}$ & $\begin{array}{c}0.02 \pm 0.3 \\
8 \\
(10) \\
\end{array}$ \\
\hline & P3(1997-99) & $\begin{array}{c}1186.86^{\mathrm{a}} \pm 9 \\
5.01(31)\end{array}$ & $\begin{array}{c}225.61 \pm 57.71 \\
(31)\end{array}$ & $\begin{array}{c}504.24 \pm 57.4 \\
5(31)\end{array}$ & $\begin{array}{c}131.35 \pm 43.05 \\
\text { (31) }\end{array}$ & $\begin{array}{l}2.24 \pm 1.20 \\
(31)\end{array}$ & $\begin{array}{c}0.09 \pm 0.29 \\
\quad(31)\end{array}$ & $\begin{array}{c}0.09 \pm 0.3 \\
3 \\
(28) \\
\end{array}$ \\
\hline & P4(2000-02) & $\begin{array}{l}1218.75^{\mathrm{ab}} \pm \\
63.92(23)\end{array}$ & $\begin{array}{c}91.71 \pm 37.73 \\
(23)\end{array}$ & $\begin{array}{c}376.66 \pm 37.5 \\
8(23)\end{array}$ & $\begin{array}{c}83.95 \pm 28.05 \\
(23)\end{array}$ & $\begin{array}{c}1.09 \pm 0.78 \\
(23)\end{array}$ & $\begin{array}{c}0.36 \pm 0.18 \\
(22)\end{array}$ & $\begin{array}{c}0.46 \pm 0.2 \\
2 \\
(19) \\
\end{array}$ \\
\hline & P5(2003-05) & $\begin{array}{c}1162.85^{\mathrm{a}} \pm 6 \\
1.91(68)\end{array}$ & $\begin{array}{c}126.56 \pm 37.18 \\
(68)\end{array}$ & $\begin{array}{c}408.99 \pm 37.0 \\
3(68)\end{array}$ & $\begin{array}{c}83.10 \pm 27.63 \\
(68)\end{array}$ & $\begin{array}{c}2.18 \pm 0.77 \\
(68)\end{array}$ & $\begin{array}{l}0.32 \pm 0.18 \\
\quad(64)\end{array}$ & $\begin{array}{c}0.59 \pm 0.2 \\
1 \\
(46)\end{array}$ \\
\hline & P6(2006-08) & $\begin{array}{c}1137.10^{\mathrm{a}} \pm 6 \\
5.39 \\
(51)\end{array}$ & $\begin{array}{c}101.17 \pm 38.54 \\
(51)\end{array}$ & $\begin{array}{c}388.35 \pm 38.3 \\
8 \\
(51) \\
\end{array}$ & $\begin{array}{c}65.48 \pm 28.66 \\
(51)\end{array}$ & $\begin{array}{c}1.96 \pm 0.80 \\
(51)\end{array}$ & $\begin{array}{c}0.46 \pm 0.19 \\
\quad(49)\end{array}$ & $\begin{array}{c}0.73 \pm 0.2 \\
3 \\
(33) \\
\end{array}$ \\
\hline & P7(2009-11) & $\begin{array}{c}1185.69^{\mathrm{a}} \pm 7 \\
3.56 \\
(35) \\
\end{array}$ & $\begin{array}{c}108.23 \pm 42.53 \\
(35)\end{array}$ & $\begin{array}{c}395.12 \pm 42.3 \\
5 \\
(35) \\
\end{array}$ & $\begin{array}{c}96.81 \pm 31.66 \\
(35)\end{array}$ & $\begin{array}{c}1.57 \pm 0.88 \\
(35)\end{array}$ & $\begin{array}{l}0.46 \pm 0.21 \\
\quad(31)\end{array}$ & $\begin{array}{c}0.73 \pm 0.2 \\
5 \\
(26) \\
\end{array}$ \\
\hline & P8(2012-14) & $\begin{array}{c}1290.43^{\mathrm{bc}} \pm \\
67.48 \\
(71) \\
\end{array}$ & $\begin{array}{c}81.38 \pm 42.03 \\
(71)\end{array}$ & $\begin{array}{c}369.63 \pm 41.8 \\
6 \\
(71) \\
\end{array}$ & $\begin{array}{c}74.00 \pm 31.28 \\
(71)\end{array}$ & $\begin{array}{c}1.59 \pm 0.87 \\
(71)\end{array}$ & $\begin{array}{l}0.44 \pm 0.21 \\
\quad(68)\end{array}$ & $\begin{array}{c}0.96 \pm 0.2 \\
5 \\
(48) \\
\end{array}$ \\
\hline \multirow{4}{*}{$\begin{array}{l}\text { Season } \\
\text { of } \\
\text { calving }\end{array}$} & Summer & $\begin{array}{c}1219.88 \pm 2 \\
2.07 \\
(96) \\
\end{array}$ & $\begin{array}{c}167.90^{\mathrm{c}} \pm 9.44 \\
(96)\end{array}$ & $\begin{array}{c}447.62^{\mathrm{c}} \pm 9.4 \\
9(96)\end{array}$ & $\begin{array}{c}108.97 \pm 6.33 \\
(96)\end{array}$ & $\begin{array}{l}2.19 \pm 0.18 \\
(96)\end{array}$ & $\begin{array}{l}0.22 \pm 0.04 \\
\quad(91)\end{array}$ & $\begin{array}{c}0.25 \pm 0.0 \\
6 \\
(67) \\
\end{array}$ \\
\hline & Rainy & $\begin{array}{c}1210.57 \pm 2 \\
8.20 \\
(54) \\
\end{array}$ & $\begin{array}{c}145.87^{\mathrm{b}} \pm 11.5 \\
5(54)\end{array}$ & $\begin{array}{c}424.02^{\mathrm{b}} \pm 11 \\
57 \\
(54) \\
\end{array}$ & $\begin{array}{c}103.27 \pm 8.05 \\
(54)\end{array}$ & $\begin{array}{l}1.87 \pm 0.22 \\
(54)\end{array}$ & $\begin{array}{c}0.25 \pm 0.05 \\
(52)\end{array}$ & $\begin{array}{c}0.37 \pm 0.0 \\
7 \\
(41) \\
\end{array}$ \\
\hline & Autumn & $\begin{array}{c}1296.07 \pm 2 \\
9.77 \\
(51) \\
\end{array}$ & $\begin{array}{c}119.85^{\mathrm{a}} \pm 12.1 \\
0(51)\end{array}$ & $\begin{array}{c}403.45^{\mathrm{a}} \pm 12 \\
12(51)\end{array}$ & $\begin{array}{l}89.92 \pm 8.50 \\
\quad(51)\end{array}$ & $\begin{array}{l}1.53 \pm 0.24 \\
(51)\end{array}$ & $\begin{array}{c}0.35 \pm 0.06 \\
(46)\end{array}$ & $\begin{array}{c}0.45 \pm 0.0 \\
8 \\
(36) \\
\end{array}$ \\
\hline & Winter & $\begin{array}{l}1244.47 \pm 1 \\
9.79(132)\end{array}$ & $\begin{array}{c}160.20^{\mathrm{bc}} \pm 8.75 \\
(132)\end{array}$ & $\begin{array}{c}441.23^{\mathrm{bc}} \pm 8.7 \\
8(132)\end{array}$ & $\begin{array}{c}101.25 \pm 5.73 \\
(132)\end{array}$ & $\begin{array}{l}2.02 \pm 0.16 \\
\quad(132)\end{array}$ & $\begin{array}{l}0.27 \pm 0.03 \\
\quad(129)\end{array}$ & $\begin{array}{c}0.34 \pm 0.0 \\
5 \\
(101) \\
\end{array}$ \\
\hline $\begin{array}{l}\text { Regressi } \\
\text { on on } \\
\text { AFC }\end{array}$ & & - & -0.051 & -0.054 & -0.019 & -0.0004 & 0.0001 & 0.0001 \\
\hline
\end{tabular}

SG- Sire Group, Figures in parenthesis are number of observations

Mean with different superscripts differ significantly among themselves

Table 2: Analysis of variance for reproduction traits

\begin{tabular}{|l|c|c|c|c|c|c|c|c|}
\hline \multirow{2}{*}{ Source of variation } & \multirow{2}{*}{ d.f } & \multicolumn{7}{|c|}{ Mean squares } \\
\cline { 3 - 9 } & 7 & AFC & FSP & FCI & NS/FCON & WP & DPR42 & DPR63 \\
\hline Sire group & $74242.85^{*}$ & 3340.11 & 3493.73 & 0.51 & 1940.22 & 0.020 & 0.137 \\
\hline $\begin{array}{l}\text { Sire within sire } \\
\text { group }\end{array}$ & 84 & 38419.28 & 4670.60 & 4727.89 & 1.72 & 2021.19 & $0.082(83)$ & $0.158(79$ \\
\hline Period & 7 & $100172.87^{* *}$ & 5309.09 & 3986.59 & 2.01 & 1959.45 & 0.047 & 0.187 \\
\hline Season & 3 & 75029.61 & $17273.54^{* *}$ & $15569.64^{* *}$ & 3.05 & 2389.13 & 0.108 & 0.192 \\
\hline Milk Yield Group & 3 & - & $53688.84^{* *}$ & $56697.00^{* *}$ & $4.28^{*}$ & $5459.03 *$ & $0.503 * *$ & $0.465^{* *}$ \\
\hline
\end{tabular}




\begin{tabular}{|l|c|c|c|c|c|c|c|c|}
\hline \multicolumn{9}{|c|}{ Table 2: Contd., } \\
\hline $\begin{array}{l}\text { Regression on AFC } \\
(\text { L) }\end{array}$ & 1 & - & $20662.52^{*}$ & $22937.11 *$ & 1.47 & 2828.40 & 0.227 & 0.059 \\
\hline Error & 227 & $\begin{array}{c}32505.67 \\
(231)\end{array}$ & 3672.60 & 3637.75 & 1.59 & 2054.35 & $\begin{array}{c}0.093 \\
(213)\end{array}$ & $\begin{array}{c}0.117 \\
(144)\end{array}$ \\
\hline
\end{tabular}

$* \mathrm{P}<0.05 ; * * \mathrm{P}<0.01$ Figures in parenthesis are degree of freedom for the respective trait

Table 3: Estimates of Heritability (Diagonal), Genetic Correlation (Above Diagonal) \& Phenotypic Correlation (Below Diagonal) Between Different Reproduction Traits

\begin{tabular}{|l|c|c|c|c|c|c|c|}
\hline & AFC & FSP & FCI & NS/FCON & WP & DPR42 & DPR63 \\
\hline AFC & $\mathbf{0 . 3 8} \pm \mathbf{0 . 1 4}$ & $0.09 \pm 0.35$ & $0.15 \pm 0.33$ & $-0.51 \pm 0.51$ & $0.11 \pm 0.16$ & $0.10 \pm 0.16$ & $-0.10 \pm 0.32$ \\
\hline FSP & $-0.02 \pm 0.05$ & $\mathbf{0 . 2 2} \pm \mathbf{0 . 2 2}$ & $0.97 \pm 0.04$ & $0.53 \pm 0.20$ & $0.49 \pm 0.27$ & $-0.88 \pm 0.22$ & $-0.84 \pm 0.19$ \\
\hline FCI & $-0.01 \pm 0.05$ & $0.96^{* *} \pm 0.01$ & $\mathbf{0 . 2 8} \pm \mathbf{0 . 1 9}$ & $0.50 \pm 0.21$ & $0.49 \pm 0.27$ & $-0.90 \pm 0.22$ & $-0.84 \pm 0.19$ \\
\hline NS/FCON & $-0.03 \pm 0.05$ & $0.52^{* *} \pm 0.04$ & $0.52^{* *} \pm 0.04$ & $\mathbf{0 . 1 0} \pm \mathbf{0 . 2 2}$ & $-0.14 \pm 0.42$ & $-0.32 \pm 0.20$ & $-0.43 \pm 0.16$ \\
\hline WP & $-0.01 \pm 0.05$ & $0.46^{* *} \pm 0.04$ & $0.44^{* *} \pm 0.05$ & $0.25^{* *} \pm 0.05$ & $\mathbf{0 . 2 2} \pm \mathbf{0 . 1 7}$ & $-0.52 \pm 0.16$ & $-0.38 \pm 0.20$ \\
\hline DPR42 & $0.10 \pm 0.05$ & $-0.70^{* *} \pm 0.04$ & $-0.68^{* *} \pm 0.04$ & $-0.41^{* *} \pm 0.05$ & $-0.42^{* *} \pm 0.05$ & $\mathbf{0 . 1 1} \pm \mathbf{0 . 1 8}$ & $0.97 \pm 0.01$ \\
\hline DPR63 & $0.04 \pm 0.06$ & $-0.71^{* *} \pm 0.04$ & $-0.67^{* *} \pm 0.04$ & $-0.41^{* *} \pm 0.05$ & $-0.32^{* *} \pm 0.05$ & $0.96^{* *} \pm 0.01$ & $\mathbf{0 . 1 3} \pm \mathbf{0 . 0 1}$ \\
$* *$ P $<0.01$ & & & & & & &
\end{tabular}

